\title{
SPECTROSCOPY OF 2-AMINOPURINE: AN MCSCF STUDY*
}

\author{
E.L. RACHOFsKY ${ }^{a}$, J.B.A. Ross ${ }^{a}$, M. KRAUSs $^{b}$ AND R. OSMAN ${ }^{c \dagger}$ \\ ${ }^{a}$ Department of Biochemistry, Mount Sinai School of Medicine \\ One Gustave L. Levy Place, New York NY 10029, USA \\ ${ }^{b}$ Center for Advanced Research in Biotechnology \\ National Institute of Standards and Technology \\ 9600 Gudelsky Drive, Rockville, Maryland 20850, USA \\ ${ }^{\complement}$ Department of Physiology and Biophysics, Mount Sinai School of Medicine \\ One Gustave L. Levy Place, New York NY 10029, USA
}

(Received July 23, 1998)

2-aminopurine is a highly fluorescent isomer of adenine that can be incorporated into DNA as a probe of structure, dynamics, and protein-DNA interactions. Interpretation of the fluorescence of 2-aminopurine in DNA requires a model of the electronic structure of this fluorophore in its ground and excited states. To this end, electronic structures and energies of the ground and lowest singlet excited states of 2-amino-9-methylpurine were calculated by the multiconfiguration self-consistent field method supplemented by multiconfiguration perturbation theory. The molecular geometry was optimized in both of these electronic states to permit investigation of both electronic excitation and fluorescence emission. The predicted energies and transition dipoles were in good agreement with experiment. The permanent molecular dipole of 2-amino-9-methylpurine increased upon excitation, suggesting that both the absorption and emission spectra should shift to slightly lower energies in polar solvents. The anomalous spectral shifts observed in water suggest that 2-aminopurine undergoes hydrogen bonding that better stabilizes the ground state than the excited state. From the calculated electrostatic potentials of these two states, the position at which this hydrogen bond forms was predicted. These results form a basis for understanding the excited states and possible intermolecular interactions of 2-aminopurine in DNA.

PACS numbers: 31.15.Ar, 31.50.+w, 33.20.Lg, 33.50.Dq

*The results of this paper were initially presented at The Jablonski Centennial Conference on Luminescence and Photophysics, July 23-27, 1998, Toruń, Poland.

tTo whom correspondence should be addressed. Tel: (212) 241-5609, fax: (212) 860-3369, e-mail: osman@inka.mssm.edu 


\section{Introduction}

Fluorescence is an invaluable tool for investigating the structure, interactions, and dynamics of biological macromolecules. This method requires that a macromolecule be labelled with one or more chromophores that have significant fluorescence quantum yield. Unlike proteins, nucleic acids rarely contain intrinsic fluorophores: the fluorescence quantum yields of the naturally occurring nitrogenous bases (adenine, guanine, cytosine, thymine, and uracil) of DNA and RNA are extremely low [1]. Consequently, fluorescence measurements of nucleic acids require labelling with an extrinsic fluorophore. One such probe is 2-aminopurine (2AP), a highly fluorescent isomer of adenine that can be incorporated into DNA with little perturbation of the normal double-helical structure. Proton NMR, molecular dynamics simulations, and denaturation studies have revealed that this analog forms an effective Watson-Crick basepair with thymine that is similar to that formed by adenine [2-4]. In addition, the lowest-energy electronic absorption of $2 \mathrm{AP}$ is shifted to longer wavelengths than those of either the natural nucleic acid bases or the aromatic amino acids, enabling selective excitation of this fluorophore from a mixture of proteins and DNA. The combination of high fluorescence quantum yield, red-shifted absorption, and minimal structural perturbation of DNA make $2 \mathrm{AP}$ an attractive probe of nucleic acid structure, dynamics, and interactions. Consequently, this fluorophore has been employed in a number of biochemical and biophysical studies [4-7].

Interpretation of the emission of $2 \mathrm{AP}$ that is incorporated into DNA is limited by an incomplete understanding of the radiative and nonradiative processes that contribute to the depopulation of the excited state. Intermolecular interactions and excited-state heterogeneity can alter either the absorption or emission spectra or the kinetics of emission decay. To develop mechanistic models of the changes in spectroscopic observables, it is necessary to characterize the ground state and excited states of the fluorophore and the possible chemical or physical interactions that these states undergo.

The photophysics of 2AP has been investigated by both experimental and theoretical methods. Holmén et al. [8, 9] utilized a combination of spectroscopic methods to resolve five electronic transitions from the observed ultraviolet absorption spectra of $2 \mathrm{AP}$ and 2-amino-9-methylpurine (2A9MP). Four of these transitions were interpreted to have $\pi \rightarrow \pi^{*}$ character and high oscillator strength; the fifth had $n \rightarrow \pi^{*}$ character and correspondingly low oscillator strength. The lowest-energy solution absorption band was assigned to a single electronic transition with $\pi \rightarrow \pi^{*}$ character. The directions of the transition dipoles for these excitations were also determined. The single transition underlying the lowest-energy band was estimated to be polarized at $+53^{\circ}$ relative to the short molecular axis (C4-C5 bond).

Several groups have measured the solvatochromic shifts in the absorption and emission spectra of $2 \mathrm{AP}$ [10-13]. In relatively non-polar solvents such as dioxane and ethyl acetate, the peak of the lowest-energy band in the absorption spectrum is found at approximately $4.11 \mathrm{eV}(302 \mathrm{~nm})$, and that of the fluorescence emission at $3.59 \mathrm{eV}(355 \mathrm{~nm})$. Both spectra were found to undergo small decreases in 
energy with increasing solvent polarity. By employing a series of solvents of varying dielectric constant and refractive index, these investigators estimated the change in the molecular dipole of the chromophore on excitation. All of these studies agreed that the dipole moment of $2 \mathrm{AP}$ increased in the excited state, although estimates of the increase by various investigators varied widely (1.6 $\mathrm{D} \leq \Delta \mu \leq 2.9 \mathrm{D})$. Notably, the spectral shifts observed in water did not follow the predictions of the classical theory of dipolar interactions. This observation led Evans et al. [13] to conclude that 2AP undergoes a specific intermolecular interaction in water, which could be hydrogen bonding. However, this hypothesis has not been tested by any direct measurement of hydrogen bond sites or affinities.

Fluorescence intensity decay kinetics can be a sensitive measure of excited-state heterogeneity and interactions. The emission decay of $2 \mathrm{AP}$ in neutral aqueous buffer is monoexponential, suggesting that under these conditions emission is from a single excited state. The intensity decay of $2 \mathrm{AP}$ that has been incorporated into oligonucleotides, however, is a complex function that cannot be represented by a single exponential $[6,7]$. This decay has typically been analyzed empirically to a multiexponential function, but a physical mechanism underlying the observed kinetics has not been established. An attempt to correlate the pre-exponential factors from fluorescence decay with ground-state conformational populations observed by proton NMR was unsuccessful [14], suggesting that the decay kinetics are at least in part a result of processes occurring in the electronic excited state. These unknown interactions highlight the importance of understanding the properties of the excited states of this fluorophore.

Previous theoretical studies of the electronic excited states of $2 \mathrm{AP}$ mostly have employed low-level semi-empirical methods [15, 16]. Only two quantum chemical studies have been published using modern methods. Holmén et al. [8] performed INDO/S calculations in an attempt to confirm the transition energies, dipoles, and moments that had been deduced from the solution spectrum of $2 \mathrm{AP}$. They obtained reasonable agreement with experiment, although the INDO/S spectrum showed significantly more transitions than could be resolved from experimental data. Unfortunately, no vapor-phase spectrum of 2AP is available to compare with their results.

The only $a b$ initio quantum chemical calculations of $2 \mathrm{AP}$ that have been published are those of Broo [17], who investigated the mechanism(s) underlying the different fluorescence quantum yields of $2 \mathrm{AP}$ and adenine. In this study, the structure of each isomer was optimized by both configuration interaction (CI) and multiconfigurational self-consistent field (MCSCF) methods in the lowest-lying singlet excited state. Adenine was predicted to buckle out of the molecular plane to a much greater extent than 2AP. Because $n \rightarrow \pi^{*}$ excitation is thought to depend on an extraplanar molecular vibration, the large difference in fluorescence quantum yield between the isomers was consequently attributed to a greater degree of vibronic coupling between $\pi \rightarrow \pi^{*}$ and $n \rightarrow \pi^{*}$ excited states in adenine than in 2AP. These results are intriguing, but must be interpreted with caution due the extremely small active space ( $4 \pi$ electrons in 4 orbitals) employed in the MCSCF calculation. It is well established that calculation of excited states of 
aromatic chromophores requires a larger active space, preferably including the entire $\pi$ manifold. In addition, the active space should include lone pair orbitals and electrons if the effects of $n \rightarrow \pi^{*}$ excitation are to be assessed.

To more thoroughly characterize the electronic spectra of this fluorophore, we have calculated the lowest-energy $\pi \rightarrow \pi^{*}$ excited state of 2A9MP using the complete active space self-consistent field (CASSCF) method supplemented by multiconfigurational second-order perturbation theory. These calculations were performed with large active spaces containing the entire $\pi$ bonding system as well as lone pairs. The geometry of the excited-state fluorophore has been optimized to obtain the emitting state. The reported solvatochromic shifts in absorption and emission spectra and the evidence suggesting hydrogen bonding are interpreted in light of the predicted molecular dipoles and electrostatic potentials of the ground and excited states of the fluorophore. The implications of these results for possible models of the emission decay kinetics of 2AP in DNA are discussed.

\section{Methods}

\subsection{Geometries}

All calculations were performed using the GAMESS program package [18]. The 6-31 $G^{*}$ basis set was employed, and core orbitals were approximated using the SBK effective core potentials for all heavy atoms [19]. The ground-state geometry was optimized using the second-order Moller-Plesset (MP2) perturbation method under the assumption of planar symmetry. The lowest singlet excited-state geometry was optimized by CASSCF gradient using two different active spaces. In the first optimization, the active space consisted of 12 electrons in $6 \pi$ and $2 \pi^{*}$ orbitals. The $6 \pi$ bonding orbitals constituted the entire aromatic conjugated system. For this calculation, the excited state was assumed to have planar symmetry, and consequently, the configurational states that could be attained from this active space only included $\pi \rightarrow \pi^{*}$ excitations. However, there are several reasons to also allow $n \rightarrow \pi^{*}$ excitations when computing the excited-state wavefunction and geometry. First, it is possible that the excited state might have some $n \rightarrow \pi^{*}$ character, which cannot be ascertained from CASSCF without one or more lone pairs in the active space. Second, geometry optimization requires methods that account for dynamic electron correlation. Inclusion of $\sigma$ orbitals in the active space couples the $\sigma$ and $\pi$ manifolds, allowing $\sigma$ electrons to respond to $\pi$ excitations. Therefore, a second excited-state geometry optimization was performed with an active space consisting of 14 electrons in $2 \sigma, 5 \pi$, and $3 \pi^{*}$ orbitals. The $\pi$ orbitals were the same as for the previous calculation, with the removal of one orbital predominantly localized to N9. The $\sigma$ orbitals were lone pairs with maximum densities at N1 and N3, respectively.

\subsection{Electronic structures, transition energies and moments}

Electronic wave functions were calculated by CASSCF for both the ground and excited states at all three geometries: the ground state and two excited states described above. The active spaces employed were the same as for the respective geometry optimizations. Molecular properties such as dipole moment and electro- 
static potential were evaluated from CASSCF wavefunctions calculated for pure states. In contrast, transition energies for absorption and emission were calculated using state-averaged CASSCF, with the two states equally weighted. State averaging has been shown to be an effective method of equalizing the effects of dynamic electron correlation on the ground and excited states [20].

To obtain more accurate transition energies from CASSCF wavefunctions, multiconfiguration quasi-degenerate perturbation theory (MCQDPT) was applied to the state-averaged CASSCF wavefunction. The active space for the multiconfiguration perturbation was in most cases the same as that for the associated CASSCF. However, in some cases, $\sigma$ orbitals were removed from the active space before perturbation. In CASSCF calculation of $\pi \rightarrow \pi^{*}$ excited states, inclusion of $\sigma$ orbitals in the active space serves to partially correct for electron correlation of the $\sigma$ electrons. Perturbation of a CASSCF wave function by MCQDPT also corrects the computed energies for the effects of dynamic correlation. Thus, $\sigma$ orbitals were removed from the active space before MCQDPT to improve the accuracy of predicted transition energies.

Transition moments for absorption and emission were calculated from the converged CASSCF wavefunctions.

\subsection{Electrostatic potentials}

Electrostatic potentials of the ground and excited states were calculated on a grid of $44 \times 52$ points of $0.25 \AA$ separation in the plane of the molecule. Contour plots were generated using the MEPMAP facility of GAMESS.

\section{Results and discussion}

Upon absorption of a photon of appropriate wavelength, a chromophore undergoes an essentially instantaneous transition from its ground state to an electronic excited state. The Franck-Condon principle (in the Born-Oppenheimer approximation) states that the rearrangement of electron density occurs without any corresponding change in the positions of nuclei. Therefore, an electronic excited state of a chromophore calculated at the ground-state geometry will be a Franck-Condon state. The energy differences between various excited states and the ground state should correspond to the observed lines in the ultraviolet absorption spectrum.

Following this instantaneous excitation, the chromophore undergoes a vibrational relaxation that stabilizes the excited state by rearranging the nuclei in response to the redistribution of electrons. This relaxation brings a fluorophore from its Franck-Condon state to the fluorescent emitting state. Therefore, the molecular geometry must be optimized in the electronic excited state to obtain the emitting state. The energy difference between this state and the ground state with the same nuclear positions should equal that of the observed fluorescence emission.

The ab initio molecular orbital calculations presented below recapitulate the cycle of excitation and emission of 2A9MP, as is summarized in Fig. 1. First, the molecular geometry was optimized in the electronic ground state. At this 


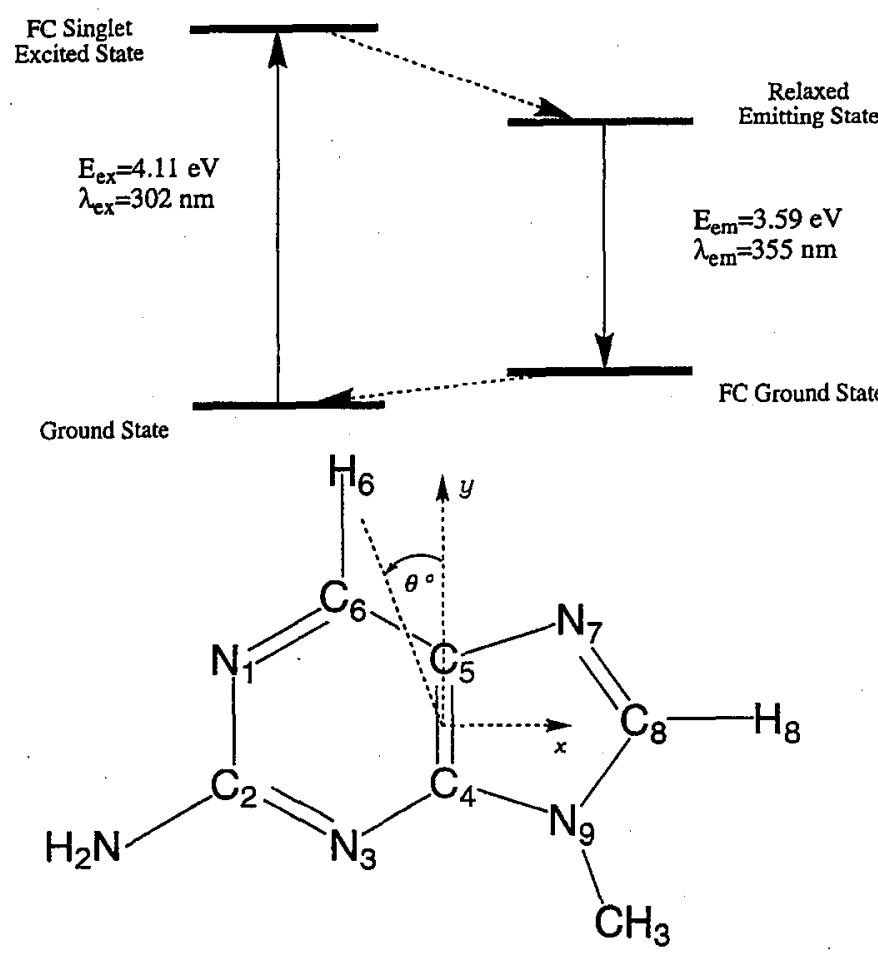

Fig. 1. (top) Jabłoński diagram for electronic excitation and fluorescence emission of 2AP. The solid arrows represent absorption and emission of a photon. The dashed arrows represent vibrational relaxation of the Franck-Condon (FC) states. The energies and wavelengths are for $2 \mathrm{AP}$ in dioxane, from Ref. [13]. (bottom) Numbering, axes, and angle conventions for $2 \mathrm{~A} 9 \mathrm{MP}$.

geometry, the lowest singlet excited state was found, and the energy of the first ultraviolet absorption predicted. The molecular geometry was then optimized in this excited state. Finally, the ground-state wavefunction was calculated at the excited-state geometry, and the energy of fluorescence emission predicted. The results are presented in four sections. In the first section, the geometries of the ground-state and excited-state chromophores are presented. In the second, the energies, transition dipoles and moments, and electronic structure changes for absorption and emission are reported. In the third section, the predicted molecular dipoles are compared to the observed solvatochromic shifts in solution spectra. In the final section, the electrostatic potentials of 2A9MP in the ground and excited states are presented, and the relative proton affinities of various functional groups are estimated.

\subsection{Geometries}

The 9-methyl derivative of 2-aminopurine was chosen for this study rather than the parent molecule $2 \mathrm{AP}$ to avoid the complications associated with tautomerism between $7-\mathrm{H}$ and $9-\mathrm{H}$ forms of $2 \mathrm{AP}$. This tautomerism has been shown 
to change the electronic structure of the chromophore [17]. However, to understand the excited state(s) of 2AP incorporated into nucleic acids, it is not necessary to consider either tautomer, since the base is 9-glycosylated in the polymer.

The numbering convention and molecular axes for 2A9MP are shown in Fig. 1. The $x$-axis 'is defined to pass through $\mathrm{C} 8$ and to bisect the N1-C2 bond. The $y$-axis is therefore approximately parallel to the C4-C5 bond. The $z$-axis is perpendicular to the molecular plane. Angles in the molecular plane are defined with respect to the $y$-axis, with a positive deflection resulting from counterclockwise rotation about the $z$-axis.

TABLE I

Bond lengths $(\AA)$ for 2A9MP.

\begin{tabular}{c|cc|c|c}
\hline \hline \multicolumn{2}{c|}{ State: } & Ground & Excited & Excited \\
\hline Bond: & Method: & MP2 & MCSCF $(8 \pi)$ & MCSCF $(2 \sigma+8 \pi)$ \\
\hline N1-C2 & & 1.38 & 1.36 & 1.37 \\
C2-N2 & 1.38 & 1.33 & 1.36 \\
C2-N3 & 1.36 & 1.36 & 1.37 \\
N3-C4 & 1.35 & 1.35 & 1.35 \\
C4-C5 & 1.43 & 1.42 & 1.43 \\
C5-C6 & 1.42 & 1.51 & 1.45 \\
C6-N1 & 1.34 & 1.36 & 1.40 \\
C5-N7 & 1.40 & 1.34 & 1.38 \\
N7-C8 & 1.34 & 1.35 & 1.30 \\
C8-N9 & 1.39 & 1.38 & 1.37 \\
N9-C1' & 1.46 & 1.46 & 1.45 \\
N9-C4 & 1.38 & 1.36 & 1.39
\end{tabular}

The ground-state geometry of 2A9MP was optimized at the MP2 level with the $6-31 G^{*}$ basis set under the condition of planar symmetry. The bond lengths for this structure are shown in Table I. These values are comparable with those from the 2AP geometry previously calculated by Broo and Holmén [21] using the same method and basis set. This planar ground-state geometry was employed for all further calculations, although in some cases no symmetry was imposed on the electronic wave function. To introduce a small degree of asymmetry, the MP2 geometry was manually perturbed by rotating $\mathrm{C} 9$ by $90^{\circ}$ around the N9-C 9 bond. This rotation did not significantly alter the properties of the calculated electronic states, and was applied only to assist in convergence of CASSCF.

The geometry of the first singlet excited state was optimized by CASSCF gradient under the condition of planar symmetry with an active space consisting of 12 electrons in $6 \pi$ and $2 \pi^{*}$ orbitals. This geometry was also optimized by CASSCF gradient without imposed symmetry with an active space consisting of 14 electrons in $2 \sigma, 5 \pi$, and $3 \pi^{*}$ orbitals, to better account for the effects of the dynamic 
correlation of the $\sigma$ electrons on the excited-state structure. The bond distances for these structures are shown in Table I.

Both excited-state geometries significantly differ from that of the ground state. Each shows increases in $\mathrm{C} 5-\mathrm{C} 6$ and $\mathrm{C} 6-\mathrm{N} 1$ bond lengths, and correspondingly shorter $\mathrm{C} 5-\mathrm{N} 7$ and $\mathrm{N} 1-\mathrm{C} 1$ bonds. The increased separation between $\mathrm{C} 6$ and the neighboring atoms is consistent with a decrease in the order of these bonds due to occupation of an anti-bonding orbital localized to $\mathrm{C} 6$. When lone pairs were included in the active space, these changes in bond order were accompanied by significant buckling of the molecule. The largest extraplanar excursions were found in the six-membered ring, with C6 more than $0.13 \AA$ above the molecular plane and N2 an equal distance below. By contrast, the heavy atoms of the five-membered ring remained virtually planar. Relaxation of planar symmetry shortened the C5-C6 bond by $0.06 \AA$, suggesting that buckling of $\mathrm{C} 6$ significantly reduced the repulsive interactions of the anti-bonding electrons at this position. The differences in the electronic wave function and energies between the two excited-state geometries are discussed in Sec. 3.4 below.

\subsection{Spectral energies and transition dipoles}

The electronic structures of the ground state and lowest singlet excited state of 2A9MP were calculated by CASSCF at all of the geometries specified above. The state-average CASSCF wave functions were subsequently subjected to MCQDPT to obtain more accurate transition energies. The results are summarized in Table II.

TABLE II

Transition energies and dipoles of 2A9MP.

\begin{tabular}{c|c|c|c|c|c|c}
\hline \hline \multirow{2}{*}{$\begin{array}{c}\text { Active } \\
\text { space }\end{array}$} & \multicolumn{3}{|c|}{ Excitation } & \multicolumn{3}{|c}{ Emission } \\
\cline { 2 - 7 } & $\begin{array}{c}E_{\mathrm{MCSCF}} \\
{[\mathrm{eV}]}\end{array}$ & $\begin{array}{c}E_{\mathrm{MCQDPT}} \\
{[\mathrm{eV}]}\end{array}$ & $\theta^{a}$ & $\begin{array}{c}E_{\mathrm{MCSCF}} \\
{[\mathrm{eV}]}\end{array}$ & $\begin{array}{c}E_{\mathrm{MCQDPT}} \\
{[\mathrm{eV}]}\end{array}$ & $\theta^{a}$ \\
\hline $12 \mathrm{e}^{-} s / 8 \pi$ & $5.69\left(218^{b}\right)$ & $4.11(302)$ & $57^{\circ}$ & $4.83(257)$ & $3.89(319)$ & $74^{\circ}$ \\
$14 \mathrm{e}^{-} s / 2 \sigma, 8 \pi$ & $5.33(233)$ & $3.89(319)$ & $99^{\circ}$ & $4.20(295)$ & $3.11(399)$ & $109^{\circ}$ \\
$14 \mathrm{e}^{-} s / 2 \sigma, 8 \pi^{c}$ & & $3.93(316)$ & & & $3.50(355)$ & \\
experiment $^{d}$ & & $4.11(302)$ & $53^{\circ}$ & & $3.50(355)$ & \\
\hline
\end{tabular}

${ }^{a}$ Transition dipole direction.

${ }^{b}$ Corresponding wavelength $(\mathrm{nm})$ for this transition energy.

${ }^{c}$ Active space for MCQDPT excluded $\sigma$ orbitals.

${ }^{d}$ From Refs. [8] and [13].

The transition energies predicted by state-averaged CASSCF for both absorption and emission were considerably greater than the experimental values. This difference was expected, since accurate prediction of the spectra of aromatic chromophores typically requires perturbation theory. The CASSCF transition energies decreased when lone pairs were included in the active space, although no configurational state functions containing $n \rightarrow \pi^{*}$ excitations had significant co- 
efficients in the CASSCF wavefunction at either the ground-state or excited-state geometry. The effect of including the lone pairs is probably due to improved correction for the correlation energy of the $\sigma$ manifold, especially in the excited state. The emission energies calculated from a particular active space were always less than the corresponding absorption energies, consistent with the expectation that optimizing the excited-state geometry should decrease the energy gap between the ground and excited states.

The MCQDPT energies of the same transitions were significantly closer to the experimental values, and again decreased upon addition of lone pairs to the active space. The excitation energy was predicted to within $0.2 \mathrm{eV}$ of the experimental peak value both with the $\pi$-only active space $\left(E_{\mathrm{ex}}=4.11 \mathrm{eV}, 302 \mathrm{~nm}\right)$ and with the more extended active space that included $2 \sigma$ orbitals $\left(E_{\text {ex }}=3.89 \mathrm{eV}\right.$, $319 \mathrm{~nm}$ ) imposed symmetry. Examination of the electronic configurations that contribute to the lowest singlet excited state indicates that it does not have significant $n \rightarrow \pi^{*}$ character. Removal of lone pairs from the perturbation calculation did not significantly alter the predicted excitation energy $(3.93 \mathrm{eV}, 316 \mathrm{~nm}), \mathrm{im}$ plying that the correlation energy of the $\sigma$ electronic manifold is approximately the same in both the ground and Franck-Condon states. The dynamic correlation of $\sigma$ electrons should not significantly change upon excitation of a planar aromatic molecule, because there is very little mixing of the $\sigma$ and $\pi$ manifolds.

The MCQDPT emission energy calculated from the excited state optimized with the $\pi$-only active space $\left(E_{\mathrm{em}}=3.89 \mathrm{eV}, 319 \mathrm{~nm}\right)$ was significantly greater than the experimental value, suggesting that this geometry did not accurately represent the fluorescent emitting state. This inaccuracy probably originates in the limitations of the CASSCF gradient for geometry optimization. Inclusion of lone pairs in the active space decreased the predicted emission energy. With lone pair electrons included in both the CASSCF and the MCQDPT active spaces, the emission energy $\left(E_{\mathrm{em}}=3.11 \mathrm{eV}, 399 \mathrm{~nm}\right)$ was significantly less than the experimental value. Removal of the lone pairs from the MCQDPT active space greatly increased the accuracy of the emission energy $(3.50 \mathrm{eV}, 355 \mathrm{~nm})$ predicted from the same state-averaged CASSCF wavefunction. At the non-planar excited-state geometry, the $\sigma$ and $\pi$ orbital manifolds are significantly mixed. Therefore, the dynamic correlation of $\sigma$ electrons is expected to increase significantly upon a $\pi \rightarrow \pi^{*}$ excitation as a consequence of the increased polarization of the $\pi$ electronic charges.

The improved accuracy of the MCQDPT emission energy calculated without imposed planar symmetry suggests that the fluorescent emitting state is significantly non-planar. In light of the high fluorescence quantum yield of 2A9MP, this result implies that excited-state buckling can occur without necessarily quenching fluorescence. Based on this improvement in emission energies with the inclusion of lone pairs in the CASSCF active space, further molecular properties will be reported only for calculations performed with this active space.

At both geometries, the excited state was found to consist predominantly of a few configurational states. The largest coefficient in each case was for a configu- 

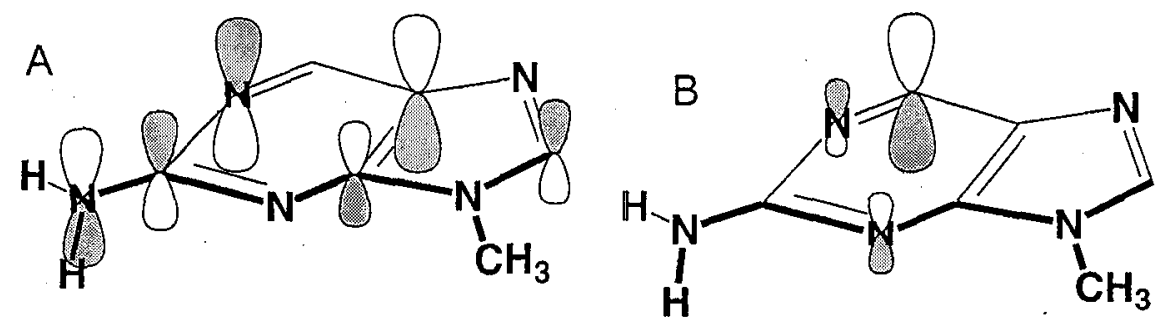

Fig. 2. Molecular orbital $p_{z}$ components at the ground-state geometry. (A) The highest occupied molecular orbital (HOMO), (B) the lowest unoccupied molecular orbital (LUMO). Only atomic orbitals with coefficients greater than 0.1 are shown.

ration representing an excitation from the highest occupied (HOMO) to the lowest unoccupied (LUMO) molecular orbitals. The $p_{z}$ coefficients of these two orbitals are depicted graphically in Fig. 2. The HOMO is a highly delocalized $\pi$ orbital, with the highest densities on C5 and N1. The LUMO is a $\pi^{*}$ orbital localized to $\mathrm{C} 6$ and N1. Thus, excitation results in net transfer of electrons to $\mathrm{C} 6$ from the other heavy atoms of the six-membered ring, including significant transfer from the extracyclic amino nitrogen. With the exception of a relatively small component of the HOMO on $\mathrm{C} 8$, the five-membered ring is not involved significantly in either excitation or emission. Occupancy of an anti-bonding orbital localized to $\mathrm{C} 6$ is consistent with the observed increases in $\mathrm{C} 5-\mathrm{C} 6$ and $\mathrm{C} 6-\mathrm{N} 1$ bond lengths in the excited state.

These results suggest that the hydrogen bonding properties of $2 \mathrm{AP}$ may change upon excitation. In the excited state, $\mathrm{N} 1$ should become a weaker hydrogen bond acceptor and N2 should become a stronger hydrogen bond donor, due to a loss of electron density at both positions. These changes may have significant consequences for the fluorescence decay of 2AP in DNA. These predictions are considered further in Sec. 3.4, in which the change in electrostatic potential upon excitation is presented.

The transition dipoles for excitation and emission predicted from these CASSCF wavefunctions are shown in Table II. The excitation dipole predicted from the $\pi$-only active space is at $57^{\circ}$ relative to the $\mathrm{C} 4-\mathrm{C} 5$ bond, while the excitation dipole predicted from the active space containing $\sigma$ orbitals is at $99^{\circ}$. The factors underlying the large difference between these results are not currently understood. The experimental excitation dipole is $53^{\circ}$, although this result has a large associated uncertainty [8]. The emission dipoles predicted from the $\pi$-only and expanded active spaces are $74^{\circ}$ and $109^{\circ}$, respectively. No experimental value of the emission dipole has been reported; however, the angle $\phi$ between the excitation and emission dipoles is related to the limiting fluorescence anisotropy $r_{0}$ by the definition $r_{0}=0.2\left(3 \cos ^{2} \phi-1\right)$. The limiting anisotropies calculated from the $\pi$-only and expanded active spaces were 0.35 and 0.38 , respectively. These results are in good agreement with the experimental value of 0.38 measured in EPA glass at $80 \mathrm{~K}[8]$. 


\subsection{Solvatochromic shifts}

The permanent molecular dipoles of $2 \mathrm{~A} 9 \mathrm{MP}$ in the ground and excited states are shown in Fig. 3. The dipoles calculated at the ground-state geometry are shown in Fig. 3A, and those at the excited-state geometry in Fig. 3B. In each of these states, the dipole is approximately parallel to the short molecular axis, and is directed toward C4. However, in the excited state, the dipole is slightly rotated toward N3 and has a greater magnitude. The dipole moment of the electronic ground state is $3.6 \mathrm{D}$ at its own geometry and $3.7 \mathrm{D}$ at the emitting geometry, respectively; the comparable values for the excited-state dipole are $4.6 \mathrm{D}$ and $4.3 \mathrm{D}$. These changes represent the increased polarization of the electronic distribution in the excited state.
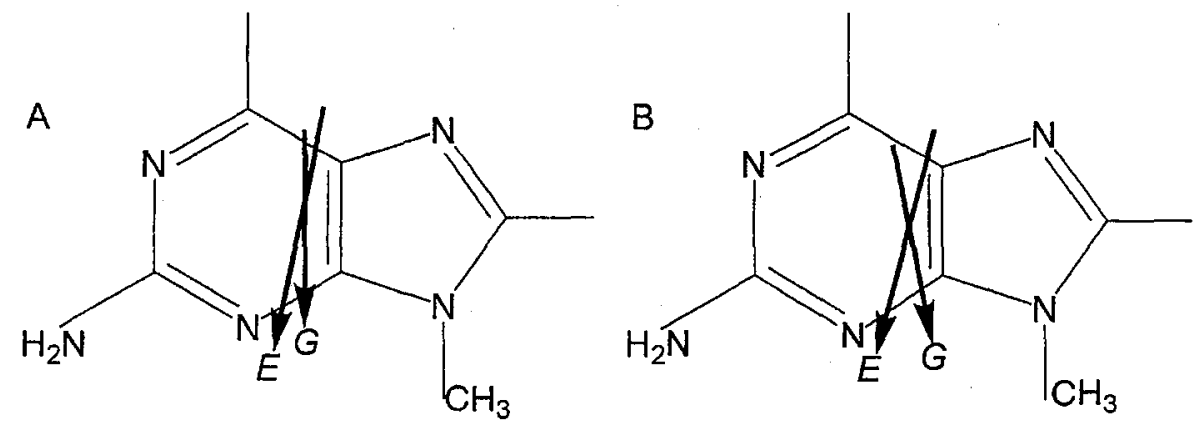

Fig. 3. Permanent molecular dipoles of 2A9MP. Electronic ground-state $(G)$ and excited-state $(E)$ dipoles at $(A)$ the ground-state geometry and $(B)$ the excited-state geometry. Size of arrows is proportional to the dipole moment.

The changes in the energy of the absorption and emission spectra of a fluorophore with changes in solvent polarity may be calculated from a classical formula [22]

$$
\Delta E=A\left(\frac{D-1}{D+2}-\frac{n^{2}-1}{n^{2}+2}\right)+B\left(\frac{n^{2}+1}{n^{2}+2}\right)
$$

where $\Delta E$ is the change in the energy center of gravity of either the absorption or emission spectrum, upon transfer from vacuum to a solvent of dielectric constant $\varepsilon$ and refractive index $n$. The constants $A$ and $B$ are given by

$$
\begin{aligned}
& A_{\mathrm{abs}, \mathrm{em}}=\frac{\mu_{\mathrm{g}, \mathrm{e}}\left(\mu_{\mathrm{g}}-\mu_{\mathrm{e}}\right)}{a^{3}}, \\
& B=\frac{\mu_{\mathrm{g}}^{2}-\mu_{\mathrm{e}}^{2}}{a^{3}},
\end{aligned}
$$

where the ground-state and excited-state dipoles are represented by $\boldsymbol{\mu}_{\mathrm{g}}$ and $\boldsymbol{\mu}_{\mathrm{e}}$, respectively. These formulae are derived from a model of the fluorophore as a dipole enclosed in a spherical cavity of radius $a$ in a continuum dielectric. The first term describes the effects of electrostatic interactions between permanent dipoles, and the second those between induced dipoles. The cavity radius for 2A9MP was 
calculated from the RHF wave function to be $7 \AA$. The coefficients $A$ and $B$ are calculated to be $-0.011 \mathrm{eV}$ and $-0.015 \mathrm{eV}$ for excitation, and $-0.009 \mathrm{eV}$ and $-0.013 \mathrm{eV}$ for emission, respectively. These small negative coefficients imply that both the absorption and emission spectra will shift slightly to lower energy (longer wavelength) as the polarity or polarizability of the solvent increases. The extent of this shift is predicted to be slightly smaller for the emission spectrum, since the difference between the dipoles is smaller at the excited-state geometry.

These energy shifts have been measured for a series of solvents [10-13]. The results of these studies are in general agreement with the predicted direction of solvatochromic shifts. However, neither absorption nor emission spectra follow the predictions of the model very closely. In water, the absorption spectrum is shifted to higher energies, suggesting that 2AP undergoes a hydrogen bond with water that stabilizes the ground state more effectively than the excited state.

\subsection{Electrostatic potentials}

To further explore how the intermolecular interactions of $2 \mathrm{~A} 9 \mathrm{MP}$ might change with excitation, the electrostatic potential of the fluorophore was calculated from the CASSCF wavefunction. Figure 4A shows the electrostatic potential for the electronic ground state at its own optimized geometry, and Fig. 4B that of the excited state at its optimized geometry. For both states, the potential surface has three local minima, situated near $N 1, N 3$, and N7, respectively. The minimum potential near $\mathrm{N} 1$ becomes $2.5 \mathrm{kcal} / \mathrm{mol}$ less negative in the excited state, while the minima near $\mathrm{N} 3$ and $\mathrm{N} 7$ become $3.2 \mathrm{kcal} / \mathrm{mol}$ and $7.2 \mathrm{kcal} / \mathrm{mol}$ more negative, respectively. Therefore, $\mathrm{N} 1$ should be a poorer hydrogen bond acceptor in the excited state, while N3 and N7 should form stronger hydrogen bonds. Based on the solvatochromic shift in the absorption spectrum, 2AP has been predicted to form a hydrogen bond with water that is stronger in the ground state than in the excited state. The changes in electrostatic potential at the three possible hydrogen bond acceptor sites are consistent with such a change in bond energy at N1, but not at N3 or N7. Therefore, it is predicted that 2AP forms a hydrogen bond at N1 in water, and that this hydrogen bond becomes weaker upon electronic excitation. It may be speculated that in DNA, the hydrogen bond between N1 of $2 \mathrm{AP}$ and thymine is weaker in the excited state. This excited-state reaction could contribute to the complex fluorescence decay kinetics of $2 \mathrm{AP}$ in DNA.

\section{Conclusions}

The electronic structures of the ground and lowest singlet excited states of 2A9MP have been calculated by CASSCF and MCQDPT. The predicted energies and transition dipoles for absorption and emission are in good agreement with experiment. The permanent molecular dipole was predicted to increase upon excitation, implying that both the absorption and emission spectra of $2 \mathrm{AP}$ should be shifted to lower energies with increasing solvent polarity. While the spectral shifts in most solvents are in agreement with this prediction, the anomalous absorption shift in water suggests that $2 \mathrm{AP}$ undergoes hydrogen bonding that better stabilizes the ground state than the excited state. The differences in the electrostatic 


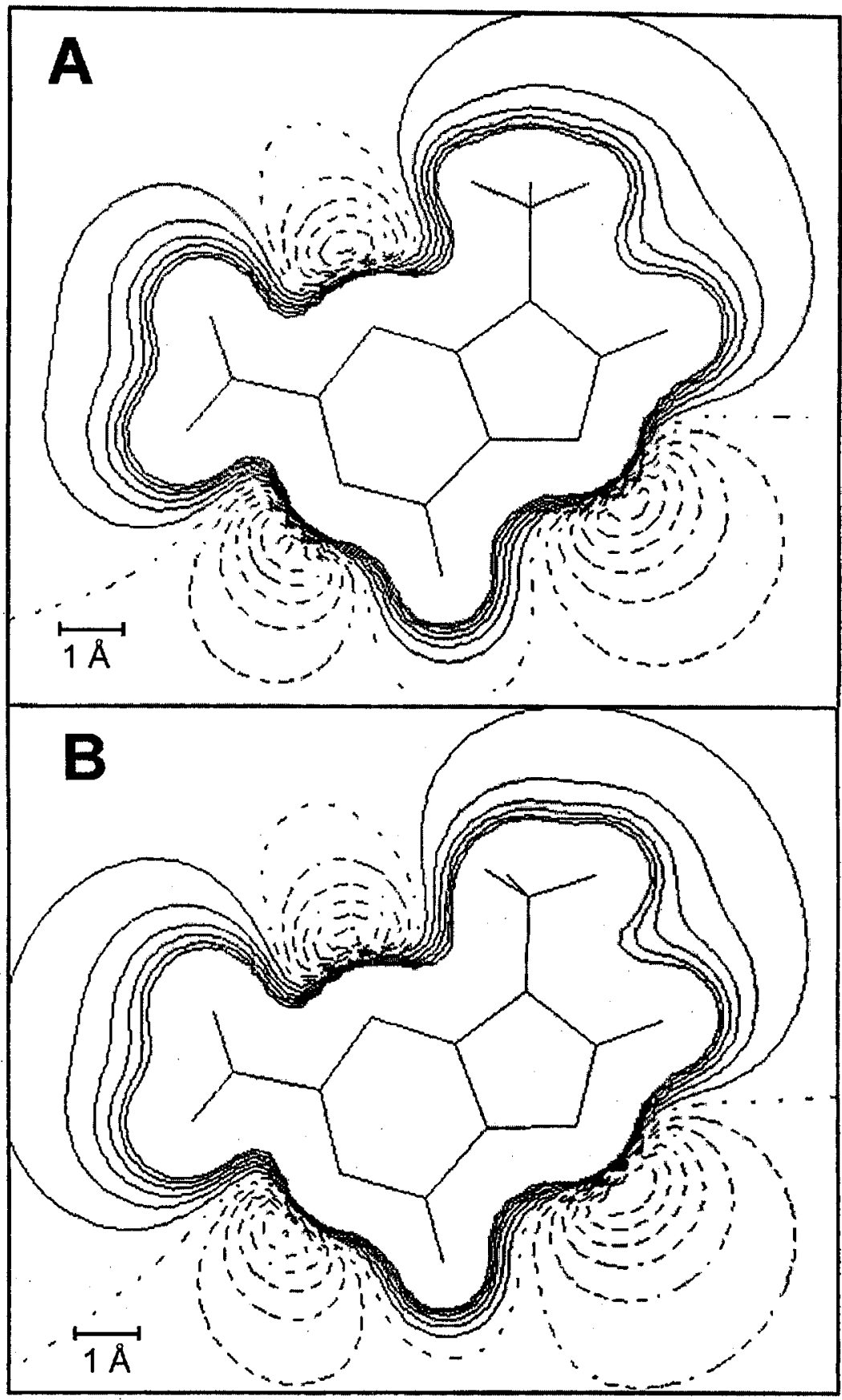

Fig. 4. Electrostatic potentials of 2A9MP. Solid lines represent positive potential, and dashed lines represent negative potential. Contours are equipotential lines separated by $10 \mathrm{kcal} / \mathrm{mol}$ each. (A) Ground state; (B) excited state. 
potential are consistent with the presence of this hydrogen bond at $\mathrm{Ni}$ but not at N3 or N7. These results imply that changes in hydrogen bonding must be considered in formulating models of 2AP fluorescence in DNA.

\section{Acknowledgment}

This work was supported by NIH grant CA63317.

\section{References}

[1] P.R. Callis, Ann. Rev. Chem. 34, 329 (1983).

[2] L.C. Sowers, G.V. Fazakerley, R. Eritja, B.E. Kaplan, M.F. Goodman, Proc. Natl. Acad. Sci. USA 83, 5434 (1986).

[3] A. Furukawa, R. Nirmala, N. Luo, R. Osman, Biophys. J. 74, A285 (1998).

[4] R.A. Hochstrasser, T.E. Carver, L.C. Sowers, D.P. Millar, Biochemistry 33, 11971 (1994).

[5] K.D. Raney, L.C. Sowers, D.P. Millar, S.J. Benkovic, Proc. Natl. Acad. Sci. USA 91, 6644 (1994).

[6] C.R. Guest, R.A. Hochstrasser, L.C. Sowers, D.P. Millar, Biochemistry 30, 3271 (1991).

[7] T.M. Nordlund, S. Andersson, L. Nilsson, R. Rigler, A. Graslund, L.W. McLaughlin, Biochemistry 28, 9095 (1989).

[8] A. Holmén, B. Nordén, B. Albinsson, J. Am. Chem. Soc. 119, 3114 (1997).

[9] A. Holmén, J. Phys. Chem. A 101, 4361 (1997).

[10] J. Smagowicz, K.L. Wierzchowski, J. Lumin. 8, 210 (1974).

[11] A. Kawski, B. Bartoszewicz, I. Gryczynski, M. Krajewski, Bull. Acad. Pol. Sci. Ser. Sci. Math. Astr. Phys. 23, 367 (1975).

[12] I. Gryczynski, A. Kawski, Bull. Acad. Pol. Sci. Ser. Sci. Math. Astr. Phys. 26, 1189 (1977).

[13] K. Evans, D. Xu, Y. Kim, T.M. Nordlund, J. Fluoresc. 2, 209 (1992).

[14] T.M. Nordlund, K.O. Evans, D. Xu, Biophys. J. 74, A21 (1998).

[15] J.S. Kwiatkowski, Acta Phys. Pol. 34, 365 (1968).

[16] I. Fischer-Hjalmars, J. Nag-Chaudhuri, Acta Chem. Scand. 23, 2963 (1969).

[17] A. Broo, J. Phys. Chem. A 102, 526 (1998).

[18] M.W. Schmidt, K.K. Baldridge, J.A. Boatz, S.T. Elbert, M.S. Gordon, J.H. Jensen, S. Koseki, N. Matsunaga, K.A. Nguyen, S. Su, T.L. Windus, M. Dupuis, J.A. Montgomery, J. Comput. Chem. 14, 1347 (1993).

[19] W.J. Stevens, H. Basch, M. Krauss, J. Phys. Chem. 81, 6026 (1984).

[20] M. Krauss, R. Osman, J. Phys. Chem. A 101, 4117 (1997).

[21] A. Broo, A. Holmén, Chem. Phys. 211, 147 (1996).

[22] D.A. Chignell, W.B. Gratzer, J. Phys. Chem. 78, 2934 (1968). 